\title{
A Fuzzy-stochastic Approach for Binary Linear Programming under Uncertainties
}

\author{
Wei Peng ${ }^{1} \&$ Rene V. Mayorga ${ }^{1}$ \\ ${ }^{1}$ Faculty of Engineering, University of Regina, Regina, Saskatchewan, Canada \\ Correspondence: Rene V. Mayorga, Faculty of Engineering, University of Regina, Regina, Saskatchewan, S4S 0A2, \\ Canada. Tel: 1-306-585-4726. E-mail: Rene.Mayorga@uregina.ca
}

Received: March 15, 2017 Accepted: May 19, 2017 Online Published: May 31, 2017

doi:10.5539/jmr.v9n3p95

URL: https://doi.org/10.5539/jmr.v9n3p95

\begin{abstract}
This paper presents an innovative Fuzzy-Stochastic Approach (FSA) to solve Binary Linear Programming (BLP) problems under uncertainties. An Interval-coefficient Fuzzy Binary Linear Programming (IFBLP) model is applied here to reflect two different types of uncertainty in a BLP problem. In the proposed IFBLP model the interval coefficient is used to reflect parameter uncertainty, and the fuzzy goal \& fuzzy constraints are used to represent model structure uncertainty. The proposed FSA would de-fuzzify the fuzzy constraints in an IFBLP model by considering its fuzzy goal; and then derive two linear BLPs with extreme crisp-coefficients from the IFBLP model, which here are called as a best optimum BLP and a worst optimum BLP. The results of the two-extreme linear BLPs are used to bound the outcome distribution of the IFBLP model. The proposed FSA is applied into a long-term traffic noise control planning to present its applicability.
\end{abstract}

Keywords: Fuzzy, Stochastic Simulation, Uncertainty, Interval-parameter, BLP

\section{Introduction}

Uncertainty is a major difficulty in solving optimization problems. It may exist in the parameters and/or in the structure in an optimization model, which are called as "parameter uncertainty" and "structure uncertainty" respectively. Previous studies show that a probability technique, interval theory, and fuzzy sets; are three methodologies for dealing with the parameter uncertainty or model structure uncertainty (Herrera et al., 1993; Zimmermann \& Pollatschek, 1984; Herrera \& Verdegay, 1991; Liu \& Sahinidis, 1997; Huang et al., 2001; Yu \& Li, 2001). In the Binary Linear Programming (BLP) area, for example, Yu and Li (2001) proposed a fuzzy method to solve a fuzzy BLP (also called as FBLP) problem, in which the model contains fuzzy coefficients in the objective function and constraints. However, their model doesn't consider the model structure uncertainty, which may result in low efficiency to solve uncertainty in the right-hand side of constraints. On the other hand, the flexible FBLP approach can efficiently solve such right-hand side uncertainty problems (Herrera et al., 1993; Zimmermann \& Pollatschek, 1984; Herrera \& Verdegay, 1991). This is because the constraints of a flexible FBLP model are expressed by fuzzy inequalities and allow some violations, which can reflect the uncertain world. However, the parameter uncertainty is not considered in their models. Huang et al. (2001) solved the parameter uncertainty problem by interval-coefficient BLP, which uses interval values (represented by a lower bound value and an upper bound value) to reflect uncertainties in model coefficients.

In this paper, an Interval-coefficient Fuzzy BLP (IFBLP) model that combines a flexible FBLP with an interval-coefficient BLP, is developed for simulating parameter uncertainty and structure uncertainty in real world cases. Because there is no existing method for solving an IFBLP model directly; an IFBLP is pre-converted into two auxiliary parametric BLP problems, which can be further dealt with by some conventional approaches (Peng \& Mayorga, 2013). Therefore, the solution of the IFBLP here roughly contains the following two major steps: (a) defuzzifying the flexible FBLP into a linear BLP to solve the fuzziness in the IFBLP, and (b) crisping the interval-coefficient BLP into a crisp BLP.

Previously, two algorithms have been developed to defuzzify a flexible FBLP. The first one searches a solution per as the concept of maximizing decision with considering both fuzzy constraint sets and a fuzzy goal (Bellman \& Zadeh, 1970; Zimmermann \& Pollatschek, 1984); the second algorithm looks for an answer based on the Representation Theorem of Fuzzy Sets without considering a fuzzy goal (Negoita \& Ralescu, 1975; Herrera \& Verdegay, 1991). However, the application of the first algorithm, called min-operator, may result in a nonlinear programming problem (see Zimmermann, 1987, pp.100-108 and 254; Herrera et al., 1993). Herrera et al. (1993) then developed an approach that used a T-norm 
operator technique to generate a single optimal alpha solution that can avoid the nonlinear problem.

There is no method that could directly crisp an interval-coefficient BLP. However, the approach developed by Chinneck and Ramadan (2000), originally designed to crisp an interval-coefficient LP, it is modified in this Paper. It is worth mentioning that the proposed approach in this Paper is not simply defuzzifying and crisping the IFBLP problems; it uses an innovative manner to deal with the problem of the infinite optimal alpha results that are caused by the integration process of the defuzzification and crisping. Moreover, a Fuzzy-Stochastic Approach (FSA) is designed to solve the high non-linear relationship between the coefficients and the optimal alpha which brought by the linear transferring process.

In detail, the proposed FSA novelty consists in: (1) utilizing the T-operator technique to defuzzify the fuzzy constraints with considering the fuzzy goal; (2) using a stochastic simulation model to find the range of optimal alpha solutions; and (3) preforming a crisping process to transfer the interval-coefficient BLP into two extreme auxiliary parametric BLPs. The IFBLP is applied into a long-term traffic noise control planning to demonstrate the applicability.

In Section 2, we present the overall FSA methodology; in particular: we introduce the formulation of IFBLP; the processes of the defuzzification and crisping; and describe the fuzzy-stochastic approach for the solution of the IFBLP. In Section 3, we demonstrate how to build an IFBLP for a long-term traffic noise control plan; show how to solve this IFBLP model; and present the results of the model a related discussion. Section 4 provides conclusions on the proposed methodology.

\section{Methodology}

\section{1 Formulation of IFBLP}

Starting from a general IFBLP model that has fuzzy constraints and interval coefficients (Peng \& Mayorga, 2013):

$$
\begin{array}{ll}
\min & f=\sum_{j \in N}\left[c_{j}^{-}, c_{j}^{+}\right] x_{j} \\
\text { s.t. } & \sum_{j \in N}\left[a_{i j}^{-}, a_{i j}^{+}\right] x_{j} \approx\left[b_{i}^{-}, b_{i}^{+}\right] \quad i \in M, \\
& x_{j} \in\{0,1\} .
\end{array}
$$

where $x_{j}$ are the binary variables; the symbols " $\widetilde{<}$ " represents fuzzy inequality, it means that the decision-maker permit some violations of the constraints; $\left[a_{i j}^{-}, a_{i j}^{+}\right] \in I(\mathfrak{R})$ represent the $\mathrm{j}$-th set of interval numbers in $\mathfrak{R}$ at the $\mathrm{i}$-th constraint, $a_{i j}^{+}$and $a_{i j}^{-}$represent the upper and lower bound coefficients, respectively; $\left[b_{i}^{-}, b_{i}^{+}\right] \in I(\Re)$ represent the set of interval numbers in $\mathfrak{R}$ on the i-th constraint, $b_{i}^{+}$and $b_{i}^{-}$are the upper and lower bounds, respectively. $\left[c_{j}^{-}, c_{j}^{+}\right] \in I(\Re)$ represent the $\mathrm{j}$-th set of interval numbers in $\mathfrak{R}$ for the objective function, $c_{j}^{+}$and $c_{j}^{-}$are the upper and lower bound coefficients, respectively.

The IFBLP model above is used to reflect two different types of uncertainty in a BLP problem: the interval coefficients reflect parameter uncertainty and the fuzzy constraints represent model structure uncertainty. To solve Model (1), two major steps are involved: 1) defuzzifying the fuzziness in the IFBLP that will result in a linear BLP, and 2) crisping the interval-coefficients that will generate a crisp BLP.

\section{2 Defuzzification Process}

Considering about a crisp coefficient BLP model with fuzzy constraints as follows:

$$
\begin{array}{ll}
\min & f=\sum_{j \in N} c_{j} x_{j} \\
\text { s.t. } & \sum_{j \in N} a_{i j} x_{j} \widetilde{<} b_{i} \quad i \in M, \\
& x_{j} \in\{0,1\} .
\end{array}
$$

The above model is also called as the flexible FBLP model. Previously, two algorithms have been developed to defuzzify the flexible FBLP model. The first one searches a solution per as the concept of maximizing decision that using the min-operator technique to connect fuzzy constraint sets and a fuzzy goal (Bellman \& Zadeh, 1970; Zimmermann \& Pollatschek, 1984); the second algorithm looks for an answer based on the Representation Theorem of Fuzzy Sets, which applying the $\alpha$-cut technique without considering a fuzzy goal (Negoita \& Ralescu, 1975; Herrera \& Verdegay, 1991). However, the application of the first algorithm may result in a nonlinear programming problem (see Zimmermann, 1987, 
pp.100-108 and 254; Herrera et al., 1993). The algorithms are introduced and discussed as follows.

\subsection{1 $\alpha$-cut Technique Approach}

We first introduce this $\alpha$-cut technique. Defining the membership functions of its ith constraint as follows:

$$
\mu_{i}(x)= \begin{cases}1 & \text { if } \sum_{j \in N} a_{i j} x \leq b_{i}, \\ \frac{b_{i}+\Delta b_{i}-\sum_{j \in N} a_{i j} x}{\Delta b_{i}} & \text { if } b_{i} \leq \sum_{j \in N} a_{i j} x \leq b_{i}+\Delta b_{i}, \quad i \in M, \\ 0 & \text { if } \sum_{j \in N} a_{i j} x \geq b_{i}+\Delta b_{i} .\end{cases}
$$

If $\forall \alpha \in(0,1]$, let any $\alpha$-cut of the constraint set becomes a classical set: $X(\alpha)=\left\{x \in \mathfrak{R} \mid \mu_{X}(x) \geq \alpha, x \in\{0,1\}\right\}$. where, $\mu_{X}(x)=\inf \left\{\mu_{i}(x), i \in M\right\}$, then Model (2) is converted into the following auxiliary parametric BLP problem:

$$
\begin{array}{ll}
\min & f=\sum_{j \in N} c_{j} x_{j} \\
\text { s.t. } & \sum_{j \in N} a_{i j} x \leq b_{i}+\Delta b_{i}(1-\alpha), \quad i \in M, \\
& x_{j} \in\{0,1\}, \alpha \in(0,1] .
\end{array}
$$

where $\Delta b_{i}$ is the tolerance of the right-hand side, and $\Delta b_{i} \geq 0 ; \alpha$ represents an intermediate variable. The major advantage of the $\alpha$-cut technique is that it ensures the fuzzy sets $\left\{\mu_{X}(x) \geq \alpha\right\}$ in convexity and boundedness. The problem is that $\alpha$ is an interval value here that requires iterative process to be generated.

\subsubsection{Min-operator Approach}

Now we discuss the Min-operator approach. For a fuzzy goal in a model, giving an aspiration value of " $f^{\prime}$ " for the objective function that desire to achieve, then Model (2) can be written as:

$$
\begin{aligned}
\text { Find } x \in\{0,1\} & \\
\text { such that } & \sum_{j \in N} c_{j} x_{j} \approx f^{\prime} \\
& \sum_{j \in N} a_{i j} x_{j} \approx b_{i} \quad i \in M .
\end{aligned}
$$

where no distinction is made between fuzzy objective and fuzzy constraints. Per as Bellman and Zadeh (1970)'s concept of maximizing decision: if the result of Model (5) will be $x^{*}$, the membership function (satisfaction degree) of the optimal decision is

$$
\mu_{D}\left(x^{*}\right)=\max _{x} \mu_{D}(x)=\max _{x} \min _{i}\left[\mu_{G}(x), \mu_{C}(x)\right]
$$

where $\mu_{G}(x)$ and $\mu_{C}(x)$ are the membership functions of the goal and the constraints; $\mu_{D}(x)$ is called as "the satisfaction degree of a decision".

In order to find the optimal value of $x^{*}$, let $\lambda=\mu_{D}(x)=\min _{i}\left[\mu_{G}(x), \mu_{C}(x)\right]$, the following model is obtained:

$\max \lambda$

$$
\begin{array}{ll}
\text { s.t. } & \lambda \leq \frac{d_{i}+\Delta d_{i}-E_{i} x}{\Delta d_{i}}, \quad i \in M+1, \\
& x \in\{0,1\}, \quad 0 \leq \lambda \leq 1 .
\end{array}
$$


where $E_{i}$ is the i-th row of $\mathrm{E}, E=\left[c, a_{1}, \ldots, a_{i}\right]^{T} \in\{\mathfrak{R}\}^{(m+1) \times n} ; d_{i}$ is the i-th row of d,$d=\left[\begin{array}{l}f^{\prime} \\ b\end{array}\right] \in\{\mathfrak{R}\}^{(m+1)}$, $\Delta d_{i}$ is the tolerance of the right-hand side, and $\Delta d_{i} \geq 0$.

The major advantage of the min-operator technique in Model (7) is that considers the goal as fuzzy. It notes that Model (1) originally has not a fuzzy goal, but be considered the goal as fuzzy when we applying the min-operator technique. However, Zimmermann pointed out that Model (7) may become a nonlinear programming problem due to nonlinear membership functions or min-operator. (see Zimmermann, 1987, pp.100-108 and 254; Herrera et al., 1993). Therefore, we need an algorithm to deal with this nonlinear problem.

2.2.3 The link of Two Methods

Herrera et al. (1993) presented an algorithm that linking above Min-operator approach and $\alpha$-cut technique for the flexible FBLP. It applies the T-norm operator technique in the process of "satisfy constraints and attain goal", which can avoid the nonlinear problem.

In order to solve Model (2) in the fuzzy solution domain $S(\alpha)$, where

$S(\alpha)=\{x \in \mathfrak{R} \mid f(x)=\min f(y), y \in X(\alpha)\}$, the following propositions are presented. The proofs relating to these propositions were given by Herrera et al. (1993)

Proposition 1 Let $\{\lambda(x)=\sup \alpha, \quad x \subset S(\alpha)\}$, and define $P(\alpha)$ is a fuzzy solution of Model (2) for each fixed $\alpha \in(0,1]$. If $\alpha^{\prime} \in(0,1]$ is a specific fixed value, and $x\left(\alpha^{\prime}\right)$ is the optimal $0-1$ solution of the corresponding problem $P(\alpha)$, then $\lambda\left(x\left(\alpha^{\prime}\right)\right)=\min \left\{\mu_{i}\left(x\left(\alpha^{\prime}\right)\right), i \in M\right\}$.

Proposition 2 Let $\theta=\lambda(x(\theta)) \in(0,1]$ and $\alpha^{\prime}=\theta+\Delta \theta$ (where $\Delta \theta=\Omega / \max \left\{\Delta b_{i}\right\}$,

and $\Omega=\left\{\begin{array}{ll}1 & \text { if } \max _{i \in M}\left\{b_{i}+\Delta b_{i}(1-\alpha)\right\}=0 \\ \text { elsewhere } & \text { els }\end{array} \min _{i \in M}\left\{b_{i}+\Delta b_{i}(1-\alpha)=\lambda\left(x\left(\alpha^{\prime}\right)\right)\right.\right.$, if $\quad \alpha^{\prime \prime}$ then $\forall \alpha \in\left(\theta, \alpha^{\prime \prime}\right]$ $x\left(\alpha^{\prime}\right)$ is an optimal value of $P(\alpha)$.

Proposition 3 Let $T(\cdot)$ to be an Archimedean t-norms operator. Using the Hamacher formulation as:

$$
H_{\gamma}\left(\mu_{A}, \mu_{B}\right)=\frac{\mu_{A} \mu_{B}}{\gamma+(1+\gamma)\left(\mu_{A}+\mu_{B}-\mu_{A} \mu_{B}\right)}, \quad \gamma>0
$$

where $\gamma$ is an arbitrary parameter. If $\gamma=1$, then $H_{\gamma}\left(\mu_{A}, \mu_{B}\right)=\mu_{A} \cdot \mu_{B}$. Therefore, the following relation holds:

$$
\sup _{\alpha} T\left(\alpha, \max _{X(\alpha)} \mu_{G}(x)\right)=\max _{x \in X} T\left(\mu_{G}(x), \mu_{C}(x)\right)
$$

Proposition 4 Let $\alpha^{*}$ is the value obtained from the Hamacher formulation (Zimmermann, 1978), and suppose $x(\alpha)$ is the optimal value of Model (2). If $\left\{x_{k}\left(\alpha_{k}\right)\right\}$ denotes the set of points of the solution $x(\alpha)$, the optimal value of $\alpha$ for Model (2) is $\alpha^{*}=\max _{\left\{x_{k}\left(\alpha_{k}\right)\right\}} T\left(\mu_{G}\left(x_{k}\left(\alpha_{k}\right)\right), \lambda\left(x_{k}\left(\alpha_{k}\right)\right)\right)$.

Based on above propositions, an iteration process for optimal value of $\alpha^{*}$ is generated, which is described in Algorithm 1 
(Herrera et al., 1993). The procedure of Algorithm 1 is shown as follows:

Algorithm 1 Let $\alpha_{1}, \alpha^{\prime}$, and $\theta$ to be three intermediate values for $\alpha$.

Step 0: let $\alpha=\alpha_{1}=\alpha^{*}=0$.

Step 1: Solve $P(\alpha)$. Let $x(\alpha)$ be an optimal value of $P(\alpha)$.

Step 2: Let $\theta=\lambda(x(\alpha))=\min \left\{\mu_{i}(x(\alpha)), i \in M\right\}$.

If $\alpha_{1}=0$, then $x(\alpha)$ is an optimal value of $P(\alpha), \forall \alpha \in\left[\alpha_{1}, \theta\right]$,

Else $x(\alpha)$ is an optimal value of $P(\alpha), \forall \alpha \in[\alpha, \theta]$.

Step 3: Let $\alpha^{\prime}=T\left(\sup \mu_{G}(x(\alpha), \theta)\right.$.

If $\alpha^{\prime}>\alpha^{*}$, then $\alpha^{*}=\alpha^{\prime}$.

Step 4: If $\theta<1$ then $\alpha_{1}=\theta, \alpha=\theta+\Delta \theta$; go to Step 1 .

Step 5: Stop.

\subsection{Crisping Process}

After defuzzifying the fuzziness, Model (1) is then transferred into an interval-coefficient BLP model with the optimum of $\alpha^{*}$.

$$
\begin{array}{ll}
\min & f=\sum_{j \in N}\left[c_{j}^{-}, c_{j}^{+}\right] x_{j} \\
\text { s.t. } & \sum_{j \in N}\left[a_{i j}^{-}, a_{i j}^{+}\right] x_{j} \leq b_{i}^{-}+\Delta b_{i}\left(1-U\left(\alpha^{*}\right)\right), \quad i \in M, \\
& x_{j} \in\{0,1\}, \alpha^{*} \in(0,1] .
\end{array}
$$

where $\Delta b_{i}=b_{i}^{+}-b_{i}^{-} ; U\left(\alpha^{*}\right)$ stand for the set of $\alpha^{*}$, each $\alpha^{*}$ in $U\left(\alpha^{*}\right)$ corresponds to a flexible FBLP model. It is evident that $U\left(\alpha^{*}\right)$ in Method (9) is boundedness that could be treated as an interval values. If we define the upper value and the lower value of a $U\left(\alpha^{*}\right)$, this interval value is identified. We give some definitions, theorems and mathematical proofs in the following sections.

\subsubsection{Value Range of $\alpha^{*}$}

Based on Proposition 4, the optimal value of $\alpha^{*}$ in a crisp BLP such as Model (4) is obtained by

$$
\alpha^{*}=\max _{\left\{x_{k}\left(\alpha_{k}\right)\right\}} T\left(\mu_{G}\left(x_{k}\left(\alpha_{k}\right)\right), \lambda\left(x_{k}\left(\alpha_{k}\right)\right)\right) \text {. }
$$

where $\left\{x_{k}\left(\alpha_{k}\right)\right\}$ indicates the set of values of the solution $x(\alpha)$.

The following Theorems, their proofs, and Algorithms have been developed by the authors for finding the value range of $\alpha^{*}$.

Theorem 1 Suppose $x(\alpha)$ is the solution value of a crisp BLP such as Model (4), $\lambda(x(\alpha))$ has a negative linear correlation relationship with the corresponding variable coefficient in the constraints.

Proof From Equation (3), we obtain $\mu_{i}^{0}(x)=\left(b_{i}+\Delta b_{i}-\sum_{j \in N} a_{i j}^{0} x_{j}\right) / \Delta b_{i}$ for Model (4). let $\alpha^{0} \in(0,1]$ is a specific fixed value, and $x\left(\alpha^{0}\right)$ is the optimal 0-1 solution of the corresponding problem $P\left(\alpha^{0}\right)$. If $\forall a_{i j}^{1} \geq a_{i j}^{0}$, then a new membership function is generated: $\mu_{i}^{1}(x)=\left(b_{i}+\Delta b_{i}-\sum_{j \in N} a_{i j}^{1} x_{j}\right) / \Delta b_{i}$. Similarly, let $\alpha^{1} \in(0,1]$ be a specific fixed value, and $x\left(\alpha^{1}\right)$ be the optimal 0-1 solution of the corresponding problem $P\left(\alpha^{1}\right)$. According to proposition 1, we have $\lambda\left(x\left(\alpha^{0}\right)\right)=\min \left\{\mu_{i}^{0}\left(x\left(\alpha^{0}\right)\right), i \in M\right\}$ and $\lambda\left(x\left(\alpha^{1}\right)\right)=\min \left\{\mu_{i}^{1}\left(x\left(\alpha^{1}\right)\right), i \in M\right\}$. Due to $x_{j} \geq 0$, we can get 
$\mu_{i}^{1}(x) \leq \mu_{i}^{0}(x)$, then $\lambda\left(x\left(\alpha^{1}\right)\right) \leq \lambda\left(x\left(\alpha^{0}\right)\right)$. Therefore, $\lambda(x(\alpha))$ has a negative linear correlation relationship with the variable coefficients in the constraints.

Theorem 2 Suppose $x(\alpha)$ is the solution of a crisp BLP such as Model (4), $\mu_{G}(x(\alpha))$ has a positive linear correlation relationship with the corresponding variable coefficient in the constraints.

Proof There are two scenarios: Min crisp BLP and Max crisp BLP.

i). For the Min crisp BLP such as Model (4):

We define $X\left(\alpha^{0}\right)=\left\{x \in \mathfrak{R} \mid \mu_{X}^{0}(x) \geq \alpha^{0}, x \in\{0,1\}\right\}$ and $X\left(\alpha^{1}\right)=\left\{x \in \mathfrak{R} \mid \mu_{X}^{1}(x) \geq \alpha^{1}, x \in\{0,1\}\right\}$, where, $\mu_{X}^{0}(x)=\inf \left\{\mu_{i}^{0}(x), i \in M\right\}$ and $\mu_{X}^{1}(x)=\inf \left\{\mu_{i}^{1}(x), i \in M\right\}$. Due to $\forall a_{i j}^{1} \geq a_{i j}^{0}$, according to Theorem 1 , we have $\lambda\left(x\left(\alpha^{1}\right)\right) \leq \lambda\left(x\left(\alpha^{0}\right)\right)$, then $\left(\min \left\{\mu_{i}^{1}\left(x\left(\alpha^{1}\right)\right), i \in M\right\} \leq \min \left\{\mu_{i}^{0}\left(x\left(\alpha^{0}\right)\right), i \in M\right\}\right)$. Therefore, $\lambda\left(x\left(\alpha^{1}\right)\right) \leq \lambda\left(x\left(\alpha^{0}\right)\right)$ is satisfied $X\left(\alpha^{0}\right) \subseteq X\left(\alpha^{1}\right)$.

As $c \cdot x\left(\alpha^{0}\right)=\min \left\{c \cdot x \mid x \in X\left(\alpha^{0}\right)\right\}$ and $x\left(\alpha^{0}\right) \in X\left(\alpha^{0}\right) \subseteq X\left(\alpha^{1}\right)$,

it follows that $c \cdot x\left(\alpha^{0}\right)=\min \left\{c \cdot x \mid x \in X\left(\alpha^{1}\right)\right\} ;$ on the other hand, $c \cdot x\left(\alpha^{1}\right)=\min \left\{c \cdot x \mid x \in X\left(\alpha^{1}\right)\right\}$ and $x\left(\alpha^{1}\right) \in X\left(\alpha^{1}\right)$, but $\forall x\left(\alpha^{1}\right) \notin X\left(\alpha^{0}\right)$. Thus, we have $c \cdot x\left(\alpha^{1}\right) \leq c \cdot x\left(\alpha^{0}\right)$.

Use the following membership function equation:

$$
\mu_{G}(x)= \begin{cases}1 & \text { if } c x \leq f^{-} \\ \frac{f^{+}-c x}{\left(f^{+}-f^{-}\right)} & \text {if } f^{-} \leq c x \leq f^{+} \\ 0 & \text { if } c x \geq f^{+}\end{cases}
$$

We have $\mu_{G}\left(x\left(\alpha^{0}\right)\right) \leq \mu_{G}\left(x\left(\alpha^{1}\right)\right)$, then $\mu_{G}(x(\alpha))$ has a positive linear correlation relationship with the corresponding variable coefficient in the constraints.

ii). For the Max crisp BLP, use an objective coefficient substitution: Let $c^{\prime}=-c$, and then convert the Max problem to a Min problem. The proof is then identical to the above proof.

According to Theorem 1, Theorem 2, and Equation (10), we obtain the following consequences, and their proofs are obvious.

(1) The value of $\alpha^{*}$ has a nonlinear relationship with the corresponding variable coefficient in the constraints.

(2) The interval coefficients will result in a continue $\alpha^{*}$ solution set.

Definition 1 For Model (9), if a value of $\alpha^{*}$ is the maximum (or minimum) in $U\left(\alpha^{*}\right)$, it is then denoted as $\alpha^{*_{+}}$(or $\alpha^{{ }^{*-}}$ ). Therefore, Model (9) can be transferred into the following model: 


$$
\begin{array}{ll}
\min & f=\sum_{j \in N}\left[c_{j}^{-}, c_{j}^{+}\right] x_{j} \\
\text { s.t. } & \sum_{j \in N}\left[a_{i j}^{-}, a_{i j}^{+}\right] x_{j} \leq b_{i}^{-}+\Delta b_{i}\left(1-\left[\alpha^{*-}, \alpha^{*+}\right]\right), \quad i \in M, \\
& x_{j} \in\{0,1\}, \alpha^{*} \in(0,1] .
\end{array}
$$

We know $\left[a_{i j}^{-}, a_{i j}^{+}\right]$and $\left[c_{j}^{-}, c_{j}^{+}\right]$are interval values with uniform distribution, but don't know the distribution type about the set of $\alpha^{* j}$ that values are between $\alpha^{{ }^{*+}}$ and $\alpha^{{ }^{*}-}$. Here, we use $\left[\alpha^{{ }^{*}-}, \alpha^{*_{+}}\right]$to indicate the value set of $\alpha^{* *}$.

\subsubsection{Solution for an Interval-coefficient BLP}

Solving a LP requires that specific values be fixed (crisped) for the coefficients in the model. An interval-coefficient contains infinite crisping coefficients, and a different coefficient will result in a different solution value for the model. Chinneck and Rammadan (2000) developed an algorithm for solving interval-coefficient LP. They declared that the best optimal result and the worst optimal result about the variables in the model can be generated by fixing the interval coefficients on their bounds. Their generalizations of the basic idea are used for the interval-coefficient BLP in this paper.

We call a specific constraint that fixing its coefficients at the lower bounds or the upper bounds as an extreme constraint. If a model contains $\mathrm{p}$ interval coefficients in the constraints, this model clearly has $2^{p+1}$ different extreme constraint combinations. let $S_{k}$ stand for the set of solutions to the k-th extreme constraint and let $S^{+}=\bigcup_{k=1}^{2^{p+1}} S_{k}$, and $S^{-}=\bigcap_{k=1}^{2^{p+1}} S_{k}$.

Definition 2 If there has one extreme constraint combination that its solution set is the same as $S^{+}$(or $S^{-}$), then it is called the maximum (or minimum) value range constraint combination.

Theorem $3 \quad$ For Model (12), suppose there are the interval constraints
$\sum_{j \in N}\left[a_{i j}^{-}, a_{i j}^{+}\right] x_{j} \leq\left[\left(b_{j}^{-}+\Delta b_{j}\left(1-\alpha^{*+}\right)\right),\left(b_{j}^{-}+\Delta b_{j}\left(1-\alpha^{*-}\right)\right)\right]$, where $i \in M, \quad$ and $\quad x_{j} \in\{0,1\} \geq 0$. Then $\sum_{j \in N} a_{i j}^{-} x_{j} \leq\left(b_{j}^{-}+\Delta b_{j}\left(1-\alpha^{*-}\right)\right)$ and $\sum_{j \in N} a_{i j}^{+} x_{j} \leq\left(b_{j}^{-}+\Delta b_{j}\left(1-\alpha^{*_{+}}\right)\right)$are the maximum value range constraint combination and the minimum value range constraint combination.

Proof Let $\sum_{j \in N} a_{i j} x_{j} \leq\left(b_{j}^{-}+\Delta b_{j}\left(1-\alpha^{*}\right)\right)$ be any legal version of the interval constraint combination, not necessarily an extreme one. Then for any particular solution $x_{j} \geq 0$ we have $\sum_{j \in N} a_{i j} x_{j} \leq \sum_{j \in N} a_{i j}^{+} x_{j}$. Therefore if $\sum_{j \in N} a_{i j}^{+} x_{j} \leq\left(b_{j}^{-}+\Delta b_{j}\left(1-\alpha^{*_{+}}\right)\right)$at $x^{*}$, then we have $\sum_{j \in N} a_{i j}^{+} x_{j} \leq\left(b_{j}^{-}+\Delta b_{j}\left(1-\alpha^{*_{+}}\right)\right) \leq\left(b_{j}^{-}+\Delta b_{j}\left(1-\alpha^{*_{-}}\right)\right)$, such that point $x^{*}$ must satisfy all possible versions of the interval constraint combination simultaneously. Therefore $\sum_{j \in N} a_{i j}^{+} x_{j} \leq\left(b_{j}^{-}+\Delta b_{j}\left(1-\alpha^{*_{+}}\right)\right)$is the minimum value range constraint combination; similarly, for any particular solution $x_{j} \geq 0$ we have $\sum_{j \in N} a_{i j}^{-} x_{j} \leq\left(b_{j}^{-}+\Delta b_{j}\left(1-\alpha^{*-}\right)\right)$ is the maximum value range constraint combination.

Theorem 4 Suppose there is the objective function $\operatorname{Min} z=\sum_{j \in N}\left[c_{j}^{-}, c_{j}^{+}\right] x_{j}$, where $x_{j} \in\{0,1\} \geq 0$. Then $\sum_{j \in N} c_{j}^{-} x_{j} \leq \sum_{j \in N} c_{j}^{+} x_{j}$ for any given solution $x^{*}$ 
Proof Since $x_{j} \geq 0$, proof is evident.

Therefore, it is not necessary to look for the distribution type of the value set of $\alpha^{*}$, we only need to know the values of $\alpha^{{ }^{*-}}$ and $\alpha^{{ }^{*+}}$.

\subsubsection{Monte Carlo Simulation}

The values of $\alpha^{*-}$ and $\alpha^{*+}$ can be found by a statistical simulation technology-Monte Carlo Simulation. Monte Carlo Simulation involves repeated generation of pseudovalues for the modeling inputs, drawn from known probability distributions within the ranges of possible values.

Algorithm 2 the Monte Carlo Simulation includes the following steps:

Step 1: generation of the pseudovalues for $\left[a_{i j}^{-}, a_{i j}^{+}\right]$and $\left[c_{j}^{-}, c_{j}^{+}\right]$, which both following the uniform distribution.

Step 2: implementation of Model (4) and Algorithm 1 with the pseudovalues to find a solution $\alpha^{*}$.

Step 3: repeat steps 1 and 2;

Step 4: compare the values of $\alpha^{*}$ in the solution set to find $\alpha^{*-}$ (lower boundary) and $\alpha^{{ }^{*}+}$ (upper boundary).

2.3.4 The best and the worst optimum models

Algorithm 3 In the interval coefficient BLP of Model (12), $x_{j} \in\{0,1\} \geq 0$. We could obtain the best optimum by solving the following BLP:

$$
\begin{array}{ll}
\min & z=\sum_{j \in N} c_{j}^{-} x_{j} \\
\text { s.t. } & \sum_{j \in N} a_{i j}^{-} x_{j} \leq\left(b_{j}^{-}+\Delta b_{j}\left(1-\alpha^{*+}\right)\right) \quad i \in M, \\
& x_{j} \in\{0,1\} .
\end{array}
$$

Furthermore, we can generate the worst optimum in Model (12) by solving the following BLP:

$$
\begin{array}{ll}
\min & z=\sum_{j \in N} c_{j}^{+} x_{j} \\
\text { s.t. } & \sum_{j \in N} a_{i j}^{+} x_{j} \leq\left(b_{j}^{-}+\Delta b_{j}\left(1-\alpha^{*-}\right)\right) \quad i \in M, \\
& x_{j} \in\{0,1\} .
\end{array}
$$

\section{Application}

Traffic noise control planning requires the decision maker to make binary decisions, it is usually considered as a BLP problem. Because high non-linear and uncertainties exist in real world cases, simply apply the BLP for a traffic noise control planning may mislead the decision maker. Here, we apply the developed IFBLP and its solution process for a long-term traffic noise control planning, to reflect uncertainty and nonlinearity in the real case and demonstrate the applicability of the proposed methodology. 


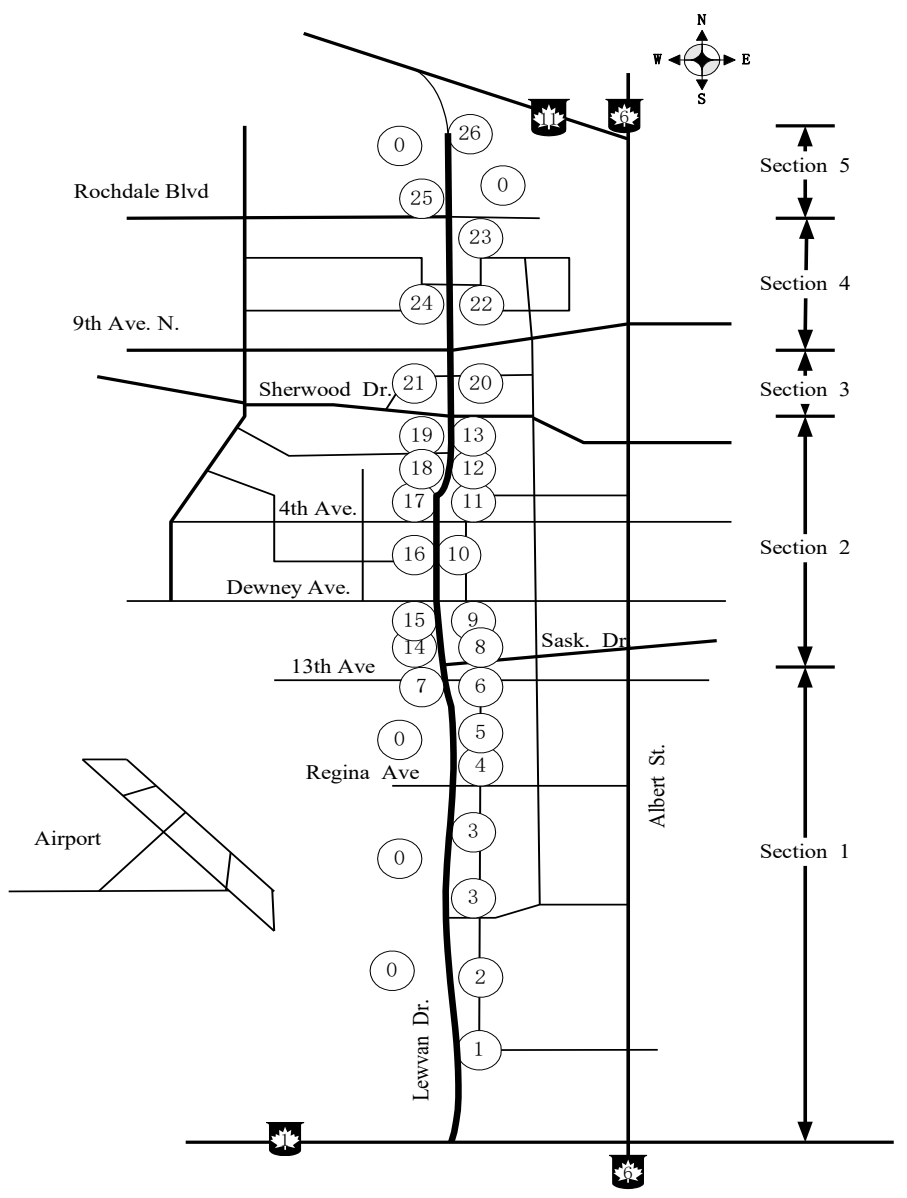

Figure 1. The studying road sketch

\subsection{Site information}

The traffic noise control site is a road of $12.4 \mathrm{~km}$ length and separated into five sections (the details are shown in Figure 1 and Table 1). There are residential zone, public zone, factory, hospital, church, school and commercial zone along this road, and traffic noise is a major complain from residents. Per as these properties and geographic information, total 26 studying zones are identified (the details are shown in Figure 1 and Table 2). We develop an IFBLP model with a 15-year planning on these 26 studying zones, and then use the proposed algorithm to solve this model and provide noise control suggestions for the decision maker.

Table 1. Five sections information

\begin{tabular}{cccccc}
\hline $\begin{array}{c}\text { Section } \\
\text { No. }\end{array}$ & $\begin{array}{c}\text { Vehicle volume } \\
\text { (vehicles/day) }\end{array}$ & $\begin{array}{c}\text { Speed } \\
(\mathrm{km} / \mathrm{hr})\end{array}$ & $\begin{array}{c}\text { Beginning } \\
\text { place }\end{array}$ & $\begin{array}{c}\text { Ending } \\
\text { place }\end{array}$ & $\begin{array}{c}\text { Length/width } \\
(\mathrm{km} / \mathrm{m})\end{array}$ \\
\hline 1 & $19,900-12,500$ & 80 & HWY\#1 & Sask. Dr. & $5.7 / 40$ \\
2 & $23,600-33800$ & 70 & Sask. Dr. & Sherw. Dr. & $3.2 / 35$ \\
3 & $19,100-19,800$ & 60 & Sherw. Dr. & $9^{\text {th }}$ Ave. N. & $1.0 / 25$ \\
4 & $22,200-26,100$ & 70 & $9^{\text {th }}$ Ave. N. & Rochd. Dr. & $1.4 / 35$ \\
5 & $6,200-$ & 100 & Rochd. Dr. & HWY\#11 & $1.1 / 40$ \\
\hline
\end{tabular}

Source: 2003 Traffic Flow Map, City of Regina 
Table 2. 26 study zones identification

\begin{tabular}{|c|c|c|c|c|c|c|c|c|c|c|c|c|c|}
\hline No & (1) & (2) & (3) & (4) & (5) & (6) & No & (1) & (2) & (3) & (4) & (5) & (6) \\
\hline 0 & Undev. & $\mathrm{U}$ & -- & -- & -- & -- & 14 & Resid. & $\mathrm{N}$ & 0.4 & 50 & $\mathrm{E}$ & 2 \\
\hline 1 & Resid. & $\mathrm{N}$ & 0.8 & 50 & $\mathrm{E}$ & 1 & 15 & Resid. & $\mathrm{N}$ & 0.35 & 30 & $\mathrm{O}$ & 2 \\
\hline 2 & Resid. & $\mathrm{N}$ & 0.5 & 80 & $\mathrm{O}$ & 1 & 16 & Resid. & $\mathrm{N}$ & 0.85 & 30 & $\mathrm{O}$ & 2 \\
\hline 3 & Recre. & A & 2.1 & -- & $\mathrm{O}$ & 1 & 17 & Church & $\mathrm{N}$ & 0.2 & 20 & $\mathrm{O}$ & 2 \\
\hline 4 & Resid. & $\mathrm{N}$ & 0.5 & 80 & $\mathrm{O}$ & 1 & 18 & Park & A & 0.2 & -- & $\mathrm{O}$ & 2 \\
\hline 5 & Recre. & A & 0.4 & -- & $\mathrm{O}$ & 1 & 19 & Resid. & $\mathrm{N}$ & 0.45 & 30 & $\mathrm{O}$ & 2 \\
\hline 6 & Resid. & $\mathrm{N}$ & 0.5 & 60 & $\mathrm{O}$ & 1 & 20 & Resid. & $\mathrm{N}$ & 0.9 & 20 & $\mathrm{O}$ & 3 \\
\hline 7 & Resid. & $\mathrm{N}$ & 0.3 & 40 & $\mathrm{E}$ & 1 & 21 & Resid. & $\mathrm{N}$ & 0.4 & 20 & $\mathrm{O}$ & 3 \\
\hline 8 & Park & A & 0.6 & -- & $\mathrm{O}$ & 2 & 22 & Resid. & $\mathrm{N}$ & 0.9 & 50 & $\mathrm{O}$ & 4 \\
\hline 9 & Hospi. & $\mathrm{Q}$ & 0.2 & 150 & $\mathrm{O}$ & 2 & 23 & Comm. & A & 0.35 & 150 & $\mathrm{O}$ & 4 \\
\hline 10 & Resid. & $\mathrm{N}$ & 0.8 & 15 & $\mathrm{O}$ & 2 & 24 & Resid. & $\mathrm{N}$ & 0.9 & 50 & $\mathrm{O}$ & 4 \\
\hline 11 & School & $\mathrm{N}$ & 0.2 & 40 & $\mathrm{O}$ & 2 & 25 & Comm. & A & 0.2 & 150 & $\mathrm{O}$ & 5 \\
\hline 12 & Park & A & 0.5 & -- & $\mathrm{O}$ & 2 & 26 & Factory & $\mathrm{S}$ & 0.5 & 150 & $\mathrm{O}$ & 5 \\
\hline 13 & Resid. & $\mathrm{N}$ & 0.6 & 20 & $\mathrm{O}$ & 2 & & & & & & & \\
\hline
\end{tabular}

Source: Air photo of Regina city, City of Regina, April 2004.

Note: (1) - Property of the studying zone,

(2) - NAC: Quiet (Q); Normal (N); Annoyance (A);Severe-Annoyance (S); Undeveloped (U)

(3) - The length of the studying zone along the road $(\mathrm{km})$

(4) - The average distance form receptor in a zone to edge of the road (m)

(5) - Berm: existing $(\mathrm{E})$; none $(\mathrm{O})$

(6) - Index of section

\subsection{Traffic Noise Control Techniques}

\subsubsection{Three Traffic Noise Control Methods}

Source control, propagation control, and receiver control are three basic traffic noise control methods. The source control is the first choice due to its proactive and it can reduce noise source for the other two methods (Herman, 1997). Meanwhile, the propagation control and the receiver control are considered as continual efforts for further mitigating if the traffic noise level still higher than the guideline at the reception sections.

Surface quality improvement of road is an effective way for the source control (shown in Table 3). Open-graded asphalt (OGA) is a desired road surface material (shown in Table 4) with a large range noise reduction up to $6.5 \mathrm{~dB}$ produced by identical vehicles that comparing with a rough surface such as Portland cement concrete (Hanson \& James, 2004). Noise barrier is the most popular noise propagation control method with noise level reduction of 5 to $10 \mathrm{dBA}$ if the barrier height is $3.5 \mathrm{~m}$. However, Noise barrier is not suit for high-rise buildings, which need insulated windows to keep noise out. To install insulated window is called the noise receiver control. 
Table 3. Noise correction factor for pavement (dBA)

\begin{tabular}{ll}
\hline Pavement Type & Noise Correction Factor \\
\hline $16 \sim 20$ mm Bitumen Seal & $+4 \sim+5$ \\
Concrete & +4 \\
$5 \sim 14$ mm Bitumen Seal & $+2 \sim+4$ \\
5 mm Bitumen Seal & $+1 \sim+2$ \\
Dense Graded Asphalt & 0 \\
Stone Mastic Asphalt & -2 \\
Boral Low Noise Asphalt & -2 \\
Open Graded Asphalt & -4 \\
\hline
\end{tabular}

Table 4. Noise reduction deterioration of OGA

\begin{tabular}{ll}
\hline Age of Surface & Noise Reduction (in $\mathrm{L}_{\mathrm{eq}}$ ) \\
\hline 0 year & $5-7 \mathrm{~dB}(\mathrm{~A})$ \\
2 years & $4-5 \mathrm{~dB}(\mathrm{~A})$ \\
4 years & $1-2 \mathrm{~dB}(\mathrm{~A})$ \\
\hline
\end{tabular}

Source: Sandberg, 1992

\subsubsection{Noise Abatement Criteria (NAC)}

Based on the noise criterion of the 23CFR772 criteria of NAC (Barry \& Reagan, 1978), traffic noise is categorized into five noise tolerances classes. They are Quiet, Normal, Annoyance, Severe annoyance, and Un-limitation. Additionally, considering the "Regulations for Community noise" (Dieter, 1995), an interval noise abatement criteria is obtained for the developed IFBLP model (shown in Table 5).

Table 5. Interval value of NAC (dBA)

\subsubsection{Traffic Noise Prediction}

\begin{tabular}{lll}
\hline Zone & Day & Night \\
\hline Quiet & $(49,55)$ & $(39,48)$ \\
Normal & $(54,59)$ & $(44,53)$ \\
Annoyance & $(58,67)$ & $(49,58)$ \\
Severe Annoyance & $(66,72)$ & $(54,63)$ \\
Unlimited & -- & -- \\
\hline
\end{tabular}

The traffic noise levels on the 26 studying zones are forecasted by a noise prediction model for the future 15 years (Peng $\&$ Mayorga, 2007). These predicted noise levels are given in interval values. Figure 2 illustrates the forecasted noise levels for zone \#11, and shows these noise levels will increase temporally in the future 15 years. Figure 3 demonstrates the forecasted noise levels on the $10^{\text {th }}$ future year for the 26 studying zones. Figure 3 demonstrates that the predicted noise levels on the $10^{\text {th }}$ future year will vary on different zones spatially.

Table 6. Road surface repaving cost and maintenance costs (1000 Dollar)

\begin{tabular}{lllllll}
\hline & Time period 1 & & Time period 2 & \multicolumn{3}{c}{ Time period 3 } \\
\hline & Repaving & Maintenance & Repaving & Maintenance & Repaving & Maintenance \\
\hline 1 & $1368-2508$ & $1026-1254$ & $1482-2622$ & $1083-1311$ & $1596-2736$ & $1140-1368$ \\
2 & $768-1408$ & $576-704$ & $832-1482$ & $608-736$ & $896-1536$ & $640-768$ \\
3 & $192-352$ & $144-176$ & $208-368$ & $152-184$ & $224-384$ & $160-192$ \\
4 & $336-616$ & $252-308$ & $364-644$ & $266-322$ & $392-672$ & $280-336$ \\
5 & $264-484$ & $198-242$ & $286-506$ & $209-253$ & $308-528$ & $220-264$ \\
\hline
\end{tabular}




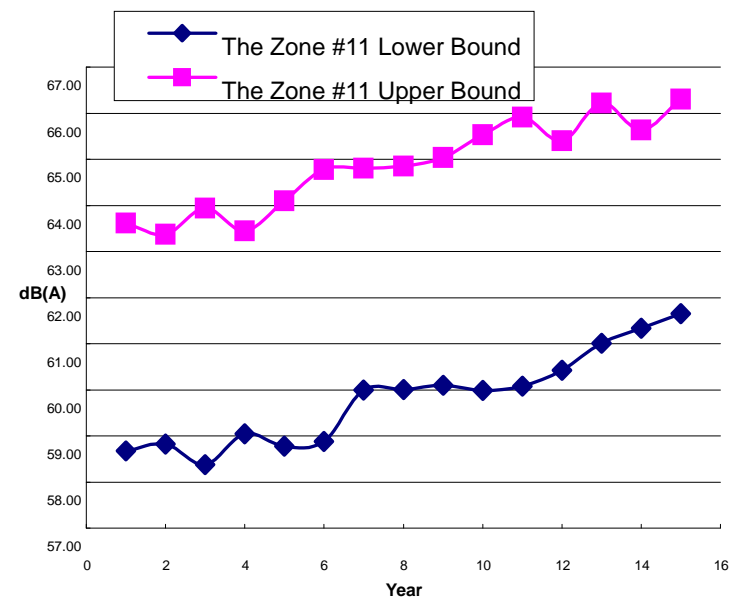

Figure 2 The predicted noise level at the zone \#11

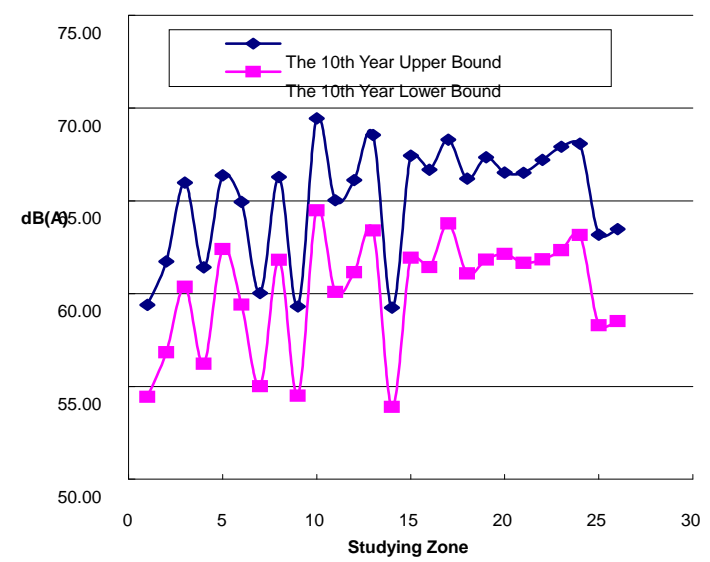

Figure 3. The predicted noise level for the future $10^{\text {th }}$ year

\subsection{IFBLP Model Building}

Based on the information described above, an IFBLP is constructed as follows:

$$
\begin{aligned}
\operatorname{Min} \mathrm{f} & =\sum_{u=1}^{U} \sum_{j=1}^{M}\left[S C_{j u}^{-}, S C_{j u}^{+}\right] x_{j u}+\sum_{u=1}^{U} \sum_{j=1}^{M}\left[S M_{j u}^{-}, S M_{j u}^{+}\right] \\
& +\sum_{c=\{0,1\}} \sum_{i=1}^{L} \sum_{k=1}^{N}\left[B C_{c i k}^{-}, B C_{c i k}^{+}\right] y_{c i k} \\
& +\sum_{c=\{0,1\}} \sum_{i=1}^{L} \sum_{k=1}^{N}\left[B M_{c i k}^{-}, B M_{c i k}^{+}\right]_{c i k}+\sum_{i=1}^{L}\left[B M D_{i}^{-}, B M D_{i}^{+}\right]
\end{aligned}
$$




$$
\begin{gathered}
+\sum_{i=1}^{L} \sum_{k=1}^{N}\left[W P A_{i k}^{-}, W P A_{i k}^{+}\right]\left[W N_{i k}^{-}, W N_{i k}^{+}\right] z_{i k} \\
+\sum_{i=1}^{L} \sum_{k=1}^{N}\left[W M A_{i k}^{-}, W M A_{i k}^{+} \llbracket W N_{i k}^{-}, W N_{i k}^{+}\right] z_{i k}+\sum_{i=1}^{L}\left[W M D_{i}^{-}, W M D_{i}^{+}\right] \\
-\sum_{u=1}^{U} \sum_{j=1}^{M}\left[S B F_{j u}^{-}, S B F_{j u}^{+}\right] x_{j u}
\end{gathered}
$$

s.t.

$$
\begin{gathered}
-\left[S R L_{i}^{-}, S R L_{i}^{+}\right] x_{j u}-\left[B R L_{k}^{-}, B R L_{k}^{+}\right] \sum_{c=\{0,1\}} \sum_{i=1}^{L} \sum_{k=1}^{N} y_{c i k} \\
-\left[W R L_{k}^{-}, W R L_{k}^{+}\right] \sum_{i=1}^{L} \sum_{k=1}^{N} y_{c i k} \approx\left[N A C_{\theta p}^{-}, N A C_{\theta}^{+}\right]-\left[N P_{\theta i k p u}^{-}, N P_{\theta i k p u}^{+}\right] \\
x, y, z \in\{0,1\} \\
\sum_{i}^{L} y_{c i k} \leq 1 \\
\sum_{i}^{L} z_{i k} \leq 1
\end{gathered}
$$

where, $(15 \mathrm{a})=$ the road surface construction and maintenance costs; $(15 \mathrm{~b})=$ the noise barrier build and maintenance costs; $(15 \mathrm{c})=$ the insulated windows installation and maintenance costs; $(15 \mathrm{~d})=$ the benefits from a new road surface; $(15 \mathrm{e})=$ the constrains of noise abatement criteria; $(15 \mathrm{f})=$ the binary constraints; $(15 \mathrm{~g})=$ only one barrier constructing on the $\mathrm{k}$ zone in the future 15 years; $(15 \mathrm{~h})=$ only one insulated window installation on the k zone in the future 15 years; (15i) $=$ no noise barrier in a commercial area or the zones already have noise barriers.

$\mathrm{i}=$ index of the year in a planning term $(i=1,2, \ldots, L) ; j=$ index of the period in a planning term $(j=1,2, \ldots M) ; t=i n d e x$ of the year in a period $\mathrm{j}(\mathrm{t}=0,1, \ldots 4) ; \mathrm{u}=$ index of the road section $(\mathrm{u}=1,2, \ldots, \mathrm{U}) ; \mathrm{k}=$ index of the studying zone $(\mathrm{k}=$ $1,2, \ldots, \mathrm{N}) ; \mathrm{p}=$ index of five classes of noise abatement criterions; $\theta=$ binary number index $(\theta=1$ represents day time; $\theta=0$ represents in night time); $\mathrm{c}=$ binary number index $(\mathrm{c}=1$ represents a commercial area or the zones of existing barrier; $\mathrm{c}=0$ represents the other areas).

$S C_{j u}^{ \pm}=$the surface repaving cost in period $\mathrm{j} ; S M_{j u}^{ \pm}=$the surface maintenance cost in the period $\mathrm{j} ; B C_{c i k}^{ \pm}=$the noise barrier constructing cost at the $\mathrm{k}^{\text {th }}$ zone in the $\mathrm{i}^{\text {th }}$ year; $B M_{c i k}^{ \pm}=$the noise barrier maintenance cost at the $\mathrm{k}^{\text {th }}$ zone in the $\mathrm{i}^{\text {th }}$ 
year; $B M D_{i}^{ \pm}=$the maintenance cost for the existing barriers at the $i^{\text {th }}$ year. $W P A_{i k}^{ \pm}=$per insulated window installing cost at $\mathrm{k}^{\text {th }}$ zone in $\mathrm{i}^{\text {th }}$ year; $W N_{i k}^{ \pm}=$the number of window at the $\mathrm{k}^{\text {th }}$ zone in the $\mathrm{i}^{\text {th }}$ year; $W M A_{i k}^{ \pm}=$per insulated window maintenance cost at the $\mathrm{k}^{\text {th }}$ zone in the $\mathrm{i}^{\text {th }}$ year; $W M D_{i}^{ \pm}=$the maintenance cost for existing insulated windows in the $\mathrm{i}^{\text {th }}$ year; $S B F_{j u}^{ \pm}=$the coefficient of benefit from renewing surface per 5 years; $N P_{\theta i k p u}^{ \pm}=$the predicted noise level (day or night) at the $\mathrm{k}^{\text {th }}$ zone on the $\mathrm{u}^{\text {th }}$ road section and belongs to the $\mathrm{p}^{\text {th }} \mathrm{NAC}$ class in the $\mathrm{i}^{\text {th }}$ year; $B R L_{k}^{ \pm}=$the reduced noise level (dBA) by barrier at $\mathrm{k}^{\text {th }}$ zone $\mathrm{dBA} ; W R L_{k}^{ \pm}=$the reduced noise level $(\mathrm{dBA})$ by insulated window at the $\mathrm{k}^{\text {th }}$ zone; $N A C_{\theta p}^{ \pm}=$the noise abatement criterions. $S R L_{t}^{ \pm}=$the reduced noise level (dBA) that benefited from OGA surface at $\mathrm{t}^{\text {th }}$ year in a period.

\subsection{IFBLP Model Solving}

Step 1 Convert the developed IFBLP model of (15) into an interval-coefficient BLP model using Algorithm 1.

$$
\operatorname{Min} \mathrm{f}=(15 \mathrm{a})+(15 \mathrm{~b})+(15 \mathrm{c})+(15 \mathrm{~d})
$$

s.t.

$$
\begin{gathered}
-\left[S R L_{i}^{-}, S R L_{i}^{+}\right] x_{j u}-\left[B R L_{k}^{-}, B R L_{k}^{+}\right] \sum_{c=\{0,1\}} \sum_{i=1}^{L} \sum_{k=1}^{N} y_{c i k} \\
-\left[W R L_{k}^{-}, W R L_{k}^{+}\right] \sum_{i=1}^{L} \sum_{k=1}^{N} y_{c i k} \\
\leq\left(N A C_{\theta p}^{-}-N P_{\theta i k p u}^{+}\right)+\left(N A C_{\theta p}^{+}-N A C_{\theta p}^{-}+N P_{\theta k p u}^{+}-N P_{\theta i k p u}^{-}\right)\left(1-U\left(\alpha^{*}\right)\right) \\
(15 \mathrm{f}),(15 \mathrm{~g}),(15 \mathrm{~h}),(15 \mathrm{i}) .
\end{gathered}
$$

Step 2 Calculate the bound values of $\alpha^{*}$ in terms of the Monte Carlo Simulation (Algorithms 2), we could obtain the values of $\alpha^{*_{-}}$and $\alpha^{*_{+}}$.

Step 3 Implement Algorithm 3, we can have the following two crisp BLP models.

1) The best optimum BLP model

$$
\begin{gathered}
\text { Min } \mathrm{f}= \\
\sum_{u=1}^{U} \sum_{j=1}^{M} S C_{j u}^{-} x_{j u}+\sum_{u=1}^{U} \sum_{j=1}^{M} S M_{j u}^{-} \\
+\sum_{c=\{0,1\}} \sum_{i=1}^{L} \sum_{k=1}^{N} B C_{c i k}^{-} y_{c i k}+\sum_{c=\{0,1\}} \sum_{i=1}^{L} \sum_{k=1}^{N} B M_{c i k}^{-} y_{c i k}+\sum_{i=1}^{L} B M D_{i}^{-} \\
+\sum_{i=1}^{L} \sum_{k=1}^{N} W P A_{i k}^{-} z_{i k} W N_{i k}^{-}+\sum_{i=1}^{L} \sum_{k=1}^{N} W M A_{i k}^{-} z_{i k} W N_{i k}^{-}+\sum_{i=1}^{L} W M D_{i}^{-}
\end{gathered}
$$




$$
-\sum_{u=1}^{U} \sum_{j=1}^{M} S B F_{j u}^{+} x_{j u}
$$

s.t.

$$
\begin{aligned}
& -S R L_{i}^{+} x_{j u}-B R L_{k}^{+} \sum_{c=\{0,1\}} \sum_{i=1}^{L} \sum_{k=1}^{N} y_{c i k}-W R L_{k}^{+} \sum_{i=1}^{L} \sum_{k=1}^{N} y_{c i k} \\
& \leq\left(N A C_{\theta p}^{-}-N P_{\theta i k p u}^{+}\right)+\left(N A C_{\theta p}^{+}-N A C_{\theta p}^{-}+N P_{\theta i k p u}^{+}-N P_{\theta i k p u}^{-}\right)\left(1-\alpha^{*-}\right)
\end{aligned}
$$

$$
\text { (15f), (15g), (15h), (15i). }
$$

2) The worst optimum BLP model

$$
\begin{gathered}
\text { Min } \mathrm{f}= \\
\sum_{u=1}^{U} \sum_{j=1}^{M} S C_{j u}^{+} x_{j u}+\sum_{u=1}^{U} \sum_{j=1}^{M} S M_{j u}^{+} \\
+\sum_{c=\{0,1\}} \sum_{i=1}^{L} \sum_{k=1}^{N} B C_{c i k}^{+} y_{c i k}+\sum_{c=\{0,1\}} \sum_{i=1}^{L} \sum_{k=1}^{N} B M_{c i k}^{+} y_{c i k}+\sum_{i=1}^{L} B M D_{i}^{+} \\
+\sum_{i=1}^{L} \sum_{k=1}^{N} W P A_{i k}^{+} z_{i k} W N_{i k}^{+}+\sum_{i=1}^{L} \sum_{k=1}^{N} W M A_{i k}^{+} z_{i k} W N_{i k}^{+}+\sum_{i=1}^{L} W M D_{i}^{+} \\
-\sum_{u=1}^{U} \sum_{j=1}^{M} S B F_{j u}^{-} x_{j u}
\end{gathered}
$$

s.t.

$$
\begin{gathered}
-S R L_{i}^{-} x_{j u}-B R L_{k}^{-} \sum_{c=\{0,1\}} \sum_{i=1}^{L} \sum_{k=1}^{N} y_{c i k}-W R L_{k}^{-} \sum_{i=1}^{L} \sum_{k=1}^{N} y_{c i k} \\
\leq\left(N A C_{\theta p}^{-}-N P_{\theta i k p u}^{+}\right)+\left(N A C_{\theta p}^{+}-N A C_{\theta p}^{-}+N P_{\theta i k p u}^{+}-N P_{\theta i k p u}^{-}\right)\left(1-\alpha^{*+}\right) \\
(15 \mathrm{f}),(15 \mathrm{~g}),(15 \mathrm{~h}),(15 \mathrm{i}) .
\end{gathered}
$$

\subsection{Results}

The related inputs are in Tables (1) to (6). The aspiration goal was designed as $f^{-}=\$ 12,000,000$ with the tolerance of $\$ 2,000,000\left(f^{+}=\$ 14,000,000\right)$. The value range of $\alpha^{*}$ was generated by Monte Carlo Simulation method. After solving the model in Matlab, the results were generated and shown in Table 7. It notes that the value range of $\alpha^{*}$ is bounded by the lower boundary of 0.313 and the upper boundary of 0.9 . The best optimal cost is $\$ 12,150,000$ and the worst optimal cost is $\$ 13,900,000$. It is found that two optimal costs are in the interval of [ $\left.f^{-}, f^{+}\right]$, where $[\$ 12,150,000, \$ 13,900,000]$ $\in[\$ 12,000,000, \$ 14,000,000]$. It is important to mention that some other optimal costs exist between two extreme optimums. These optimal costs are discrete because they are generated by the binary variables in the IFBLP model, and they can be used as trade off by decision maker. 
Table 7. Results of IFBLP model

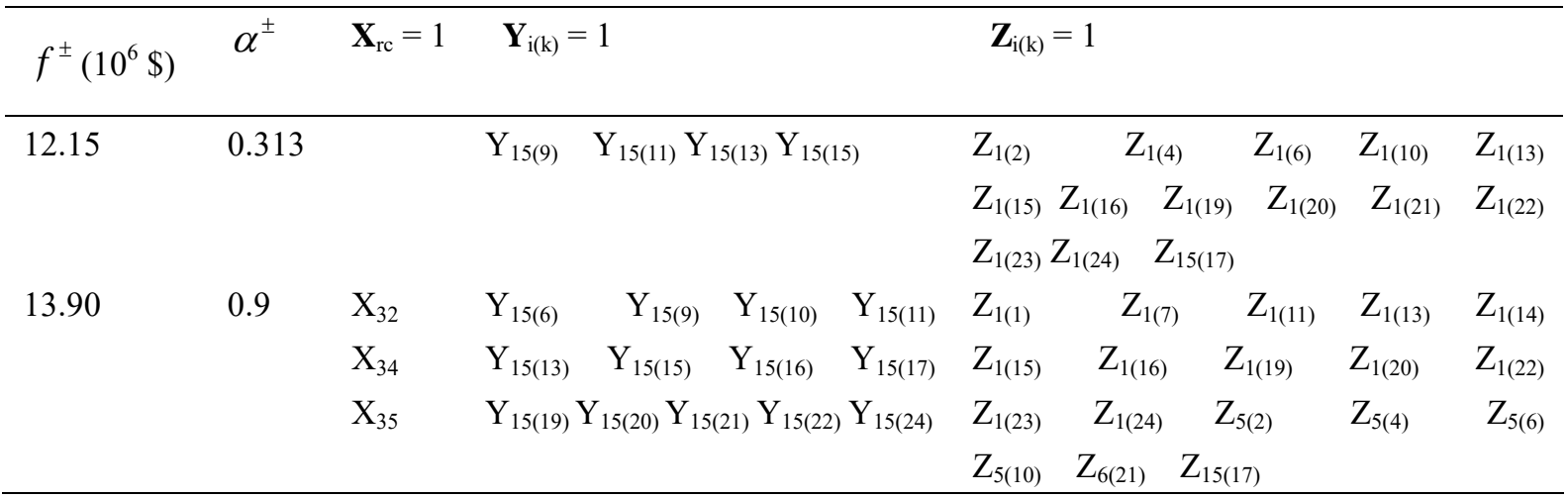

Note: $\mathrm{X}=1$ means Road Surface Updating, $\mathrm{Y}=1$ means Barrier Building, $\mathrm{Z}=1$ means Insulted Window Installing,

Period index $r=1,2,3$; Section index $c=1, \ldots 5$., Year index $i=1, \ldots 15$; Zone index $k=1, \ldots 26$.

Additionally, it is found that the optimal cost results of the developed IFBLP model are highly related to the aspiration goal and its tolerances. For instance, if the aspiration goal is set at a low value and its tolerance is small, the solution domain will be reduced, and then a feasible solution may not be obtained. therefore, the aspiration goal and tolerance need to be pre-design thought this is time consuming.

\section{Conclusions}

In this paper, an IFBLP model is developed to represent binary linear problems under uncertainties. The fuzzy constraints and interval coefficients are designed in the developed IFBLP model, in which the interval coefficients reflect parameter uncertainty and the fuzzy goal \& fuzzy constraints represent model structure uncertainty. The solution of the FSA for the IFBLP was generated, having the following novelies: (1) utilized the T-operator technique to defuzzify the fuzzy constraints with considering the fuzzy goal; (2) used a stochastic simulation model to find the range of optimal alpha solutions; and (3) preformed a crisping process to transfer the interval-coefficient BLP into two extreme auxiliary parametric BLPs. In other words, the developed algorithm ensures a linear transformation in the defuzzification process; ensures to find a single $\alpha^{*}$ solution for a flexible FBLP; and ensures to identify the boundaries of $\alpha{ }^{*}$ in an interval-coefficient BLP. Through the best optimum BLP model and the worst optimum BLP model, a closed set of optimal costs could be generated for the IFBLP model. This proposed IFBLP model was applied into a long-term traffic noise control planning. After implemented the developed FSA solution, the results yielded are used to demonstrate its applicability.

\section{Acknowledgment}

This paper research has been supported by a grant (No: 155147-2013) from the Natural Sciences and Engineering Research Council of Canada (NSERC).

\section{References}

Barry, T. M., \& Reagan, J. A. (1978). FHWA-RD-77-108FHWA. highway traffic noise prediction model, U. S. Department of Transportation. Washington DC

Bellman, R. E., \& Zadeh, L. A. (1970). Decision making in a fuzzy environment. Management Science, 17, $141-164$. https://doi.org/10.1287/mnsc.17.4.B141

Chinneck, J. W., \& Ramadan, K. (2000). Linear programming with interval coefficients. Journal of the Operational Research Society, 51, 209-220. https://doi.org/10.1057/palgrave.jors.2600891

Dieter, G. (1995). Regulations for Community Noise. Noise/News International.

Hanson, D. I., \& James, R. S. (2004). Colorado DOT tire/pavement noise study. Report No. CDOT-DTD-R-2004

Herrera, F., \& Verdegay, J. L. (1991). Approaching fuzzy integer linear programming problems, in: M. Fedrizzi, J. Kacprzyk \& M. Roubens, Eds., Interactive Fuzzy Optimization. Springer-Verlag, Berlin, 78-91. https://doi.org/10.1007/978-3-642-45700-5_9

Herrera, F., Verdegay, J. L., \& Zimmermann, H. -J. (1993). Boolean programming problems with fuzzy Constrains. European Journal of Operation Research, 55, 285-293. https://pdfs.semanticscholar.org/cf63/58876fd759290870bc6d15b8060206eef00c.pdf

Herman, L. A. (1997). Analysis of strategies to control traffic noise at the source. Transportation Research Record 1626 
paper no. 97-1519, 41-48. http://dx.doi.org/10.3141/1626-05

Huang, G. H., Sae-Lim, N., Chen, Z., \& Liu, L. (2001). Long-term planning of waste management system in the City of Regina - An integrated inexact optimization approach. Environmental Modeling \& Assessment 6, $285-296$. https://doi.org/10.1023/A:1013394118863

Liu, M. L., \& Sahinidis, N. V. (1997). Process planning in a fuzzy environment. European Journal of Operation Research, 100, 142-169. https://doi.org/10.1016/S0377-2217(96)00025-2

Peng, W., \& Mayorga, R. V. (2013). An interval-coefficient fuzzy binary linear programming, the solution, and its application under uncertainties. Journal of Operation Research Society (JORS), 64(10), 1557-1569. https://doi.org/10.1057/jors.2012.111

Peng, W, \& Mayorga, R. (2007). Assessing traffic noise impact based on probabilistic and fuzzy approaches under uncertainty. Stoch. Environ. Res. Risk Assess., 22(4), 541-550. https://doi.org/10.1007/s00477-007-0173-7

Negoita, C. V., \& Ralescu, D. A. (1975). Applications of Fuzzy Sets to Systems Analysis. Birkhauser, Stuttgart. https://doi.org/10.1007/978-3-0348-5921-9

Yu, C. -S., \& Li, H. -L. (2001). An algorithm for generalized fuzzy binary linear programming problems. European J of Operation Research, 133, 496-511. https://doi.org/10.1016/S0377-2217(00)00193-4

Zadeh, \& B. R. Gaines, Eds., Fuzzy Sets and Decision Analysis. North-Holland, New York, 133-145.

Zimmermann, H. -J., \& Pollatschek, M. A. (1984). Fuzzy 0-1 linear programs, in: H. -J. Zimmermann, L. A.

Zimmermann, H. -J. (1987). Fuzzy Sets, Decision Making, and Expert Systems. Kluwer Academic Publishers, Boston. https://doi.org/10.1007/978-94-009-3249-4

Zimmermann, H. -J. (1991). Fuzzy Set Theory and its Applications. 2nd edn. Kluwer Academic Publishers, Boston. https://doi.org/10.1007/978-94-015-7949-0

Zimmermann, H. -J. (1978). Fuzzy programming and linear programming with several objective functions.

\section{Copyrights}

Copyright for this article is retained by the author(s), with first publication rights granted to the journal.

This is an open-access article distributed under the terms and conditions of the Creative Commons Attribution license (http://creativecommons.org/licenses/by/4.0/). 